\title{
An Inverse Dynamic Approach for Quantitative Muscle Force Estimation during Human Standing-Up Process*
}

\author{
Enguo CAO**,***, Yoshio INOUE***, Tao LIU*** and Kyoko SHIBATA*** \\ **School of Design, Jiangnan University, 1800 Lihu Avenue, Wuxi, 214122, China \\ ***Department of Intelligent Mechanical Systems Engineering, Kochi University of Technology \\ 185 Miyanokuchi, Tosayamada-Cho, Kami-City, Kochi 782-8502, Japan \\ E-mail: ceg24942849@foxmail.com
}

\begin{abstract}
As it is inconvenient to directly measure tension forces of muscles attaching on limbs, an inverse dynamic approach for quantitative muscle force estimation during human standing-up process was developed. In the standing-up experiment, a rehabilitation robot was used for offering assistance and measuring dynamic parameters of body segments. Ground reaction force (GRF) and center of pressure (COP) of human body, rotational motions of trunk, thigh and shank were real-time measured by the sensors of the robot system. Meanwhile, the AnyBody Modeling System was adopted for calculating muscle forces of lower limbs. In AnyBody Modeling System, a musculoskeletal model composed of thigh, shank, foot, four joints and fifteen muscles was developed. The GRF, COP and motion data measured with sensors were imported into the model, and then the tension forces of muscles of lower limb were calculated through an inverse dynamics method. Furthermore for the validation of the rehabilitation experiment, the activation levels of muscles were also directly measured by an electromyography (EMG) system, and the calculated AnyBody results matched the measured EMG results. Therefore, this muscle force estimation approach appears to be practical for determining muscle forces in the musculoskeletal analysis of human limbs.
\end{abstract}

Key words: Inverse Dynamic, Muscle Force, Standing-Up, AnyBody, EMG

\section{Introduction}

Currently, many countries are experiencing the phenomenon of population aging. Physical degeneration among aged persons can result in deterioration of lower limb motor function. Furthermore, stroke or brain injuries have become common afflictions among the aged, resulting in an increasing number of hemiplegics ${ }^{(1)}{ }^{(2)}$. Under current medical conditions, a large proportion of those affected cannot recover completely, and rehabilitation training has become one of the most workable methods of treatment in many situations. As a result, motor function recovery activities have aroused widespread interest and the effectiveness of muscle strength training equipment has been greatly advanced in many countries ${ }^{(3-5)}$. Moreover in the evaluation of rehabilitation activities, better understanding of the working of human lower limb muscles is needed for medical diagnosis, for designing rehabilitation training machines, and making wearable artificial limbs. Therefore, it is valuable to develop a musculoskeletal model of human lower limbs for supporting the improvement of rehabilitation machines, and an easy-operating muscle force estimation method for evaluating the recovery effectiveness in clinical applications.

*Received 14 Apr., 2012 (No. 12-0181) [DOI: 10.1299/jbse.8.63]

Copyright $(\odot 2013$ by JSME 
Human lower limbs constitute a complex musculoskeletal system. For direct measurement method of muscle force, electromyography (EMG) method is becoming more accepted in muscle analysis of human limbs. Despite the fact that many researchers still have reservations about the EMG technique; it has been already frequently applied as a standard clinical tool in identifying activation levels in human muscle. However, in the EMG method the muscle activity result is relatively large or small, expressed in unit of \%, rather than quantitative Newton values ${ }^{(6)}{ }^{(7)}$. Therefore, as direct measurement of the surface and inner muscles in lower limbs is not easy, the quantitative muscle forces have been estimated via indirect methods ${ }^{(8)}{ }^{(9)}$, and the method of calculating muscle forces with dynamic models was introduced ${ }^{(10-11)}$. But for the calculating method, the acquirement of motion information of limbs and forces information applied on limbs is necessary, and the construction of a musculoskeletal dynamic model is essential. Furthermore, the accurate model may advance more precise muscle force results than the simplified model ${ }^{(12)}$.

In the field of human body segment motion estimation, the commercial motion camera system is the most popular instrument and the standard tool for measuring movement of limbs, but it is expensive for applications and difficult to utilize in a real-time controlled program ${ }^{(13)}$. By contrast, wearable sensor systems based on miniature type force sensors, acceleration sensitive units and gyroscopes have aroused more attention in more various situations due to their lower costs and easy-operating characteristics ${ }^{(14-16)}$. In the field of musculoskeletal dynamic modeling, the AnyBody Modeling System, which concerns on musculoskeletal kinematic analysis and works in a minimum fatigue criterion way, can be employed in various applications of variable situations. Furthermore, quantitative results of muscle forces can be calculated through an inverse dynamics method in this system ${ }^{(17)}$.

In this paper, a quantitative approach for estimating muscle forces only by measuring the angular motions and ground reaction force of lower limb was developed. A rehabilitation robot was used for offering assistance and measuring dynamic parameters during the standing-up process. In the rehabilitation activities, rotational motions of trunk, thigh and shank were measured by three wearable motion sensors, and ground reaction force (GRF) and center of pressure (COP) were measured by a miniature type force plate. In AnyBody Modeling System, a lower limb musculoskeletal model including thigh, shank, foot, four joints and fifteen muscles was developed, and the sensor-measured motion and force data of lower limb were imported into the model. Then quantitative muscle forces of lower limbs were calculated using an inverse dynamics analysis method. Furthermore, for validating the muscle force results, the EMG method was adopted in the standing-up experiment and the muscle activation levels were directly measured.

\section{Materials and Methods}

\subsection{Rehabilitation Robot and Sensor System}

As shown in Fig.1 (a), a double rope rehabilitation robot was developed for training human lower limb motor functions. The level of assisting force provided by the robot is adjusted according to users' intention while assuring the adequate movement trajectory of body trunk. The assist force is real-time controlled by the back rope. The back rope can decide whether to offer assist forces or just follow the user's movement while maintaining the rope tension, that because the sensor system and the algorithm controller can recognize the intended movement of the user. The back rope moves up faster when the user owns his/her own strength to stand up, moves up slower when the user needs help, or even moves down at a safe speed if the user intends to sit down. This ability ensures the rehabilitation system can spend more time on training the weak position of the user, and increase the recovery effect of the system. Meanwhile, the movement trajectory is real-time controlled by the front rope. As shown in Fig. 1 (b), paired lengths of front rope and back rope decide the movement trajectory of point $\mathrm{C}$, so while the length of back rope is being decided, the 
trajectory of point $\mathrm{C}$ can be controlled by adjusting the length of front rope. As a research result in our laboratory, the desirable movement trajectories to different height of users were saved in the control program. So while the length of back rope is being decided by the assist force and real-time measured by sensor, an adequate normal trajectory of the user can be controlled by real-time adjusting the length of front rope in the control program. An impedance control method is developed to control two servo motors (MR-J2S), and the motors are connected directly with the front and back ropes. The rope tensile forces are real-time measured by two high precision load cells fixed on the ropes, and the rope lengths are real-time measured by a photoelectric encoder in the motors. In rehabilitation training activities, the GRF and COP are real-time measured by a ground reaction force sensor, and angular motions of trunk, thigh, and shank are real-time measured by three wearable sensors constituted with accelerometers and gyroscopes. Additionally the whole hardware system is connected and controlled by a computer program system ${ }^{(18)}$.

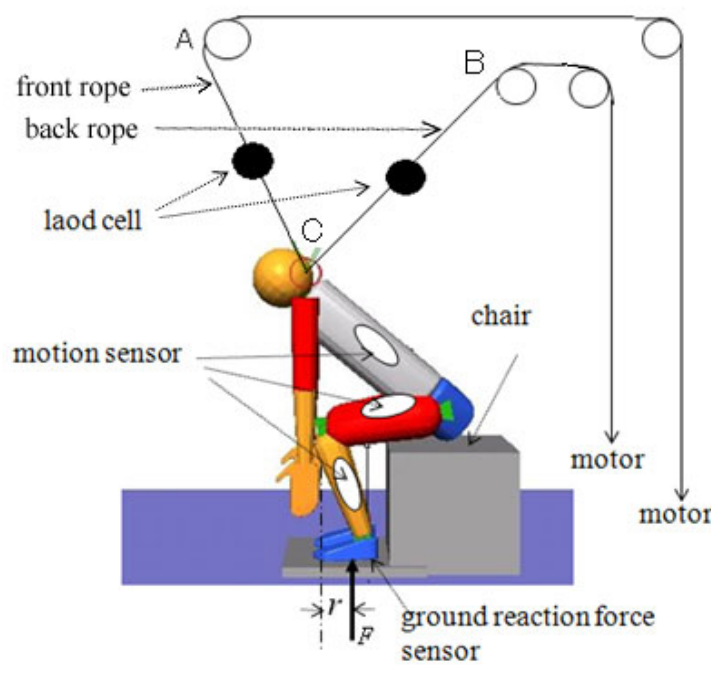

(a) Standing-up rehabilitation robot

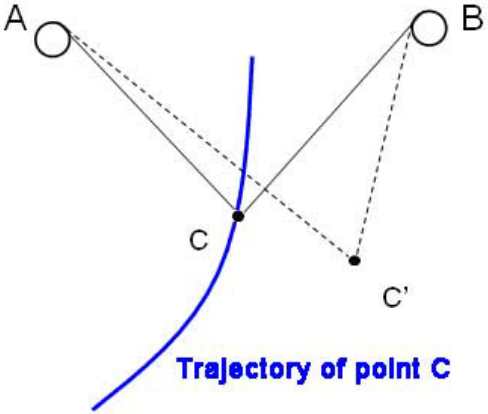

(b) Principle of the double rope system

Fig. 1 Schematic diagram of the standing-up rehabilitation robot.

\subsection{Musculoskeletal Model in AnyBody Modeling System}

The AnyBody Modeling System is not only a professional musculoskeletal modeling system, but also a kinematics and kinetics analysis system, and in the system the inverse dynamics method is adopted for estimating muscle forces. In human body, muscles are activated by the central nervous system (CNS) based on a complicated electro-chemical process. Determining the activation that realizes a desired movement requires an extremely intricate control algorithm. AnyBody imitates the workings of the CNS by computing backwards from the movement and load specified to the necessary muscle forces in a process known as inverse dynamics. Maximum synergism would be the case where all muscles capable of a positive contribution to balancing the external load work together, in such a way that the maximum relative load on any muscle in the system is as small as possible. So in AnyBody a minimum fatigue criterion way is employed because fatigue is likely to occur first in the muscle working on the maximum relative load, it makes physiological sense that the body might work that way. It means that the body would maximize its endurance and precision, this criterion might decide survival of the fittest in an environment where organisms are competing with each other for limited resources.

In AnyBody system environment a two-dimensional musculoskeletal model of human lower limb was created, and the geometric structure of the model was decided based on the human anatomy datum. As shown in Fig. 2, because the Vastus Medialis (VM), Vastus 
Lateralis (VL), and Vastus Intermedius (VI) own similar locations in the coordinate plane, in the model one resultant muscle is built in the front of thigh to simulate the muscles of VM\&VL\&VI. For the same reason, another two resultant muscles are built in the shank to simulate the muscles of Flexor Hallucis Longus \& Flexor Digitorum Longus (FHL\&FDL), and Extensor Hallucis Longus \& Extensor Digitorum Longus (EHL\&EDL). To sum up, thirteen muscles are involved in the model, they are Psoas Major (PM), Gluteus Maximus (GM), Vastus Rectus (VR), VM\&VL\&VI, Biceps Femoris (BF), Anterior Tibialis (AT), Gastrocnemius (GAST), Soleus (SOL), Peroneus Longus (PL), Peroneus Brevis (PB), Posterior Tibialis (PT), FHL\&FDL, EHL\&EDL, and all the muscles are built with the maximum strength of 5000 N. As shown in Fig. 3, the coordinates of revolute joints of hip, knee, ankle, toe and muscle joint points are determined by measuring datum of the human lower limb ${ }^{(19)}$. To make the model suit to different individuals, a size factor is indicated based on the height of users. Furthermore, the setting of fixed hip joint avoids the dynamic affects produced by the rehabilitation robot, and makes GRF be the only external load on the model.

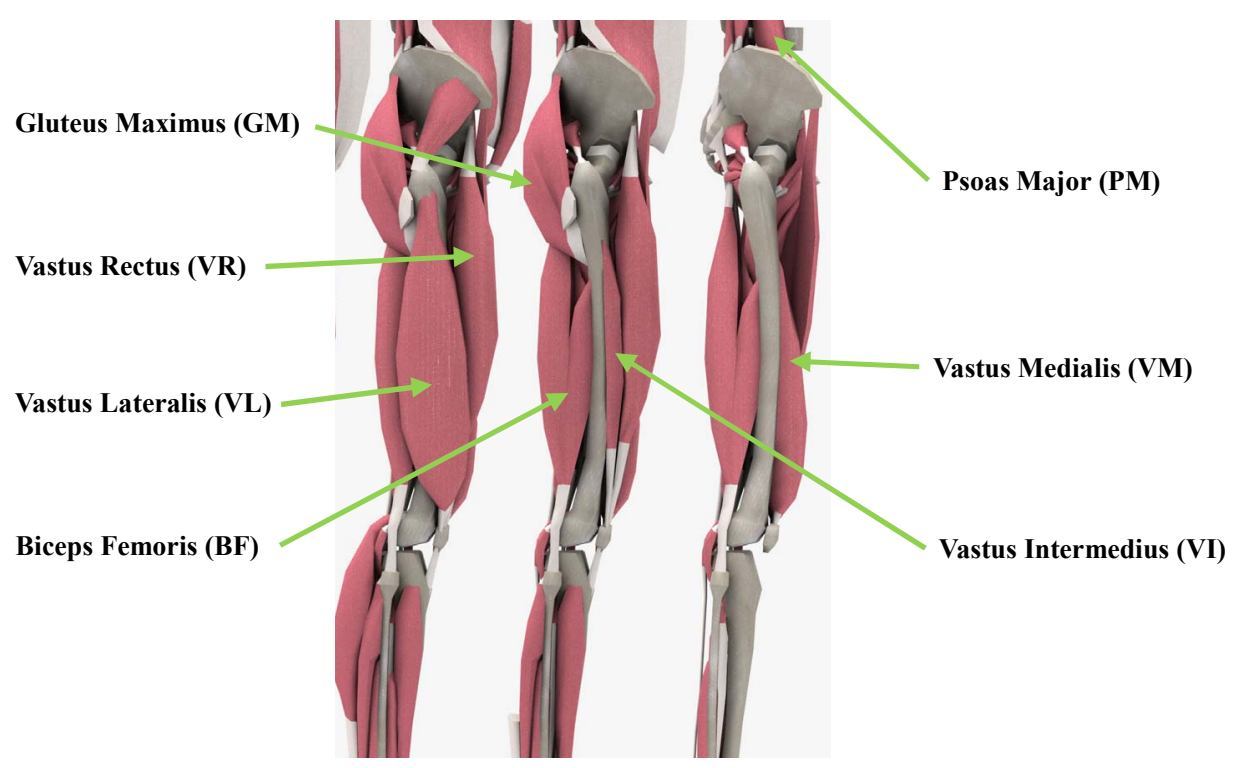

(a)

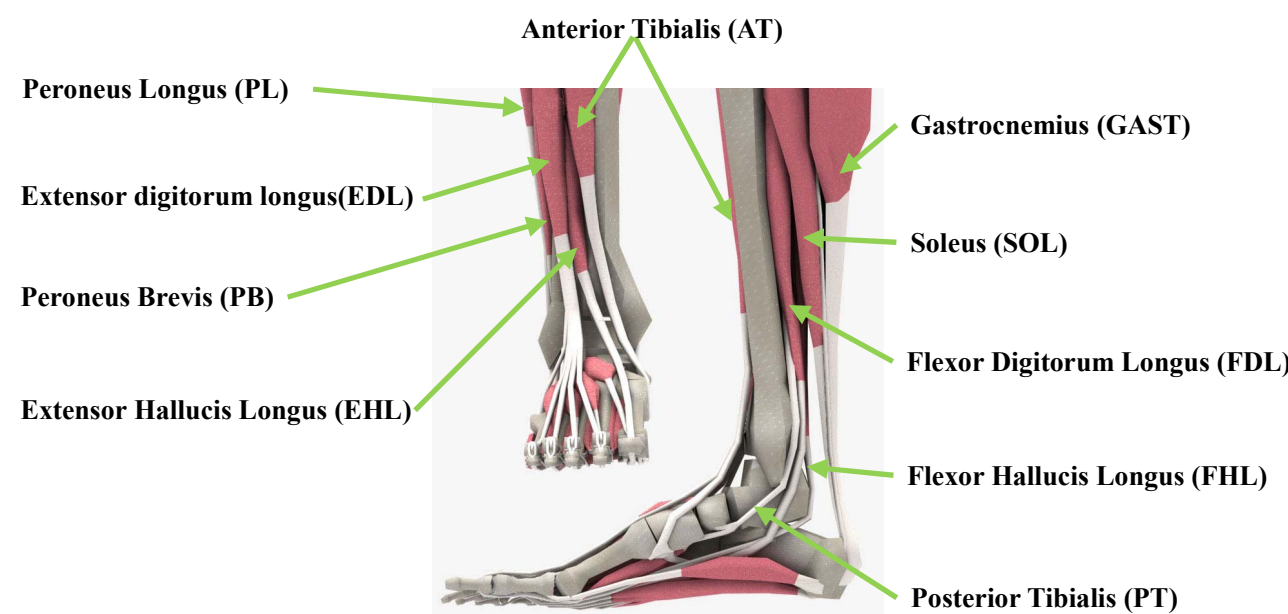

(b)

Fig. 2 Schematic diagram of muscles included in the AnyBody model.

(a) Muscles of thigh.

(b) Muscles of shank and foot. 


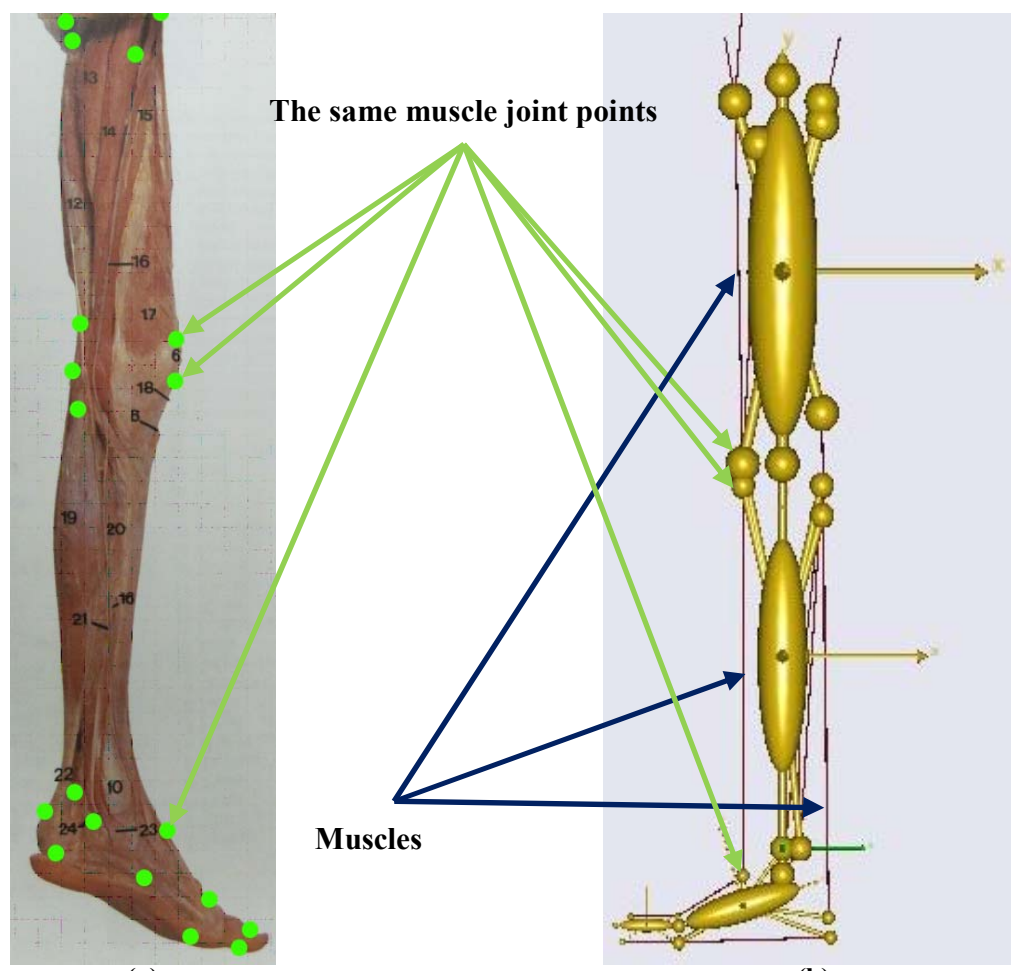

(a) (b)

Fig. 3 Method for obtaining coordinates of elements on AnyBody model.

(a) Human anatomy model for obtaining geometric data of human lower limb.

(b) Musculoskeletal model in AnyBody Modeling System, whose coordinates were determined based on measuring geometric data of (a).

\subsection{Calculation of Muscle Force by Importing Measured Data into AnyBody System}

For calculating muscle forces of lower limb, the sensor measured motion and GRF data were imported into the AnyBody Modeling System. As shown in Fig. 4, the rotation angles of lower limb are clarified. Because each angular motion data $\left(\phi_{\text {thigh }}, \phi_{\text {shank }}, \phi_{\text {foot }}\right)$ are measured in general coordinate system and all the parts in the model are regarded as rigid bodies, the relative angular motion data ( $\Delta \phi_{\text {thigh }}, \Delta \phi_{\text {shank }}, \Delta \phi_{\text {foot }}$ ) can be calculated by the quantitative subtraction. Furthermore, in the standing-up process we assume that no relative movement occurs on the toe joint and the value of $\phi_{\text {foot }}$ is regard as zero all the time. Therefore, the relative movement of thigh, shank, and foot in the model are driven by the angular data of $\Delta \phi_{\text {thigh }}, \Delta \phi_{\text {shank }}, \Delta \phi_{\text {foot }}$, which are calculated by the equation (1), (2), and (3) respectively.

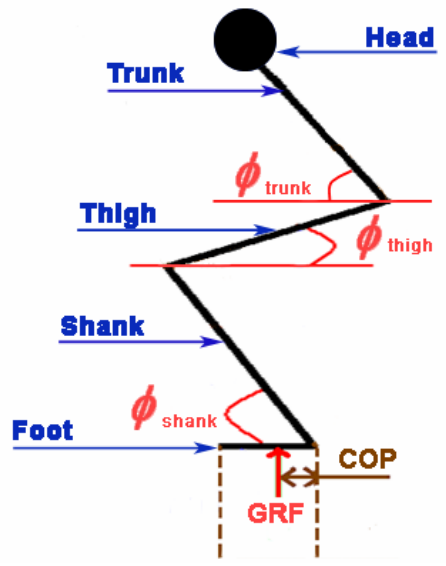

Fig. 4. Diagram for illustrating dynamic parameters of the human body. 


$$
\begin{aligned}
& \Delta \phi_{\text {thigh }}=\phi_{\text {thigh }} \\
& \Delta \phi_{\text {shank }}=\phi_{\text {shank }}-\phi_{\text {thigh }} \\
& \Delta \phi_{\text {foot }}=\phi_{\text {foot }}-\phi_{\text {shank }}
\end{aligned}
$$

To simulate the true situation of standing-up process, one group of GRF and COP data is transferred into two groups of force data, and applies on two loading points located on the bottom plane of foot model. This transformation can perfectly imitate the GRF variation and COP shifting because the foot model is regarded as a rigid body system, and in this way the whole external force process is conducted. The model works as an integrated kinetics system after all bone units are combined by joints and muscles, and the quantitative muscle forces are calculated through the inverse dynamic analysis method.

\subsection{Validation of Muscle Force Result by EMG Method}

To validate the calculated muscle force results of AnyBody Modeling System, the EMG method was adopted to directly measure muscle activation level of VM\&VL\&VI, AT, GAST and SOL. A personal-EMG system (P-EMG-0403A01) includes hard type sensors, a filter box and a data processing system. The raw EMG results measured by hard type sensors is filtered and rectified by the filter box and the data processing system into integral EMG results, which represent the muscle activation levels. One hundred percent standard voluntary contraction $(100 \% \mathrm{SVC})$ is defined as the standard isolation of muscle activity in the respective muscle tests for normalization of the EMG signal ${ }^{(20)}$. In the data processing system, both the raw EMG and the integral EMG are real-time recorded and displayed. Furthermore, the integral EMG results are used to validate the muscle force results calculated in AnyBody Modeling System.

\section{Experimental Study}

\subsection{Experiment Method}

Test experiments were designed to validate the effectiveness of the muscle force estimation approach on four subjects (age: $25 \pm 3$ years, height: $170 \pm 5 \mathrm{~cm}$, mass: $61 \pm 12 \mathrm{~kg}$ ) who have no history of musculoskeletal pathology or injury. As shown in Fig. 5, the subjects were requested to stand up from a chair at self-selected speed using, respectively, own-standing method without assistance and impedance control method with the assistance of the rehabilitation robot. Furthermore in the impedance control experiment the subjects hypothesized that they owned not enough strength to stand up independently. In the start position, the subject's elbow joints were in contact with the homologous knee joints, while in the terminal position the subject's legs were straight. In the experiment the subjects were attached to the rehabilitation robot by a conjunction jacket, and they were requested to keep their feet on the force plate. The three wearable motion sensors were fixed by belts on the trunk, thigh, and shank respectively. Precise locations of the motion sensors attached to limbs are unnecessary because all limb segments are regarded as rigid segments. While the subjects were standing up, the angular motions of trunk, thigh, and shank were real-time measured with the unit of degree $\left({ }^{\circ}\right)$. And the GRF and COP were real-time measured with the unit of, respectively, Newton $(\mathrm{N})$ and millimeter $(\mathrm{mm})$. The EMG method was adopted to directly measure muscle activation levels of VM\&VL\&VI, AT, GAST and SOL, and the measured data were real-time recorded by the personal-EMG application system.

The next step was importing the motion and GRF data into AnyBody Modeling System for the calculation of muscle forces. In AnyBody system, the measured GRF and COP data were transformed into two groups of force data on two locations, that imitated GRF variation and COP shifting perfectly. In addition, the motion data $\left(\Delta \phi_{\text {thigh }}, \Delta \phi_{\text {shank }}, \Delta \phi_{\text {foot }}\right)$ were imported into the model to drive the movement of the body segments. As shown in Fig. 
6, in AnyBody system environment the standing-up process and the muscle activation process were demonstrated visually in a easy-understood way, and the muscle forces and joint moments were calculated through the inverse dynamics method while lower limb muscles collaborated with each other in the standing-up process. Before the experiment, the objective and method of the experiment were explained to the subjects, and their written and oral consent to the experiment was obtained. This experiment had been pre-approved by the ethics committee of the Department of Intelligent Mechanical System Engineering, Kochi University of Technology.

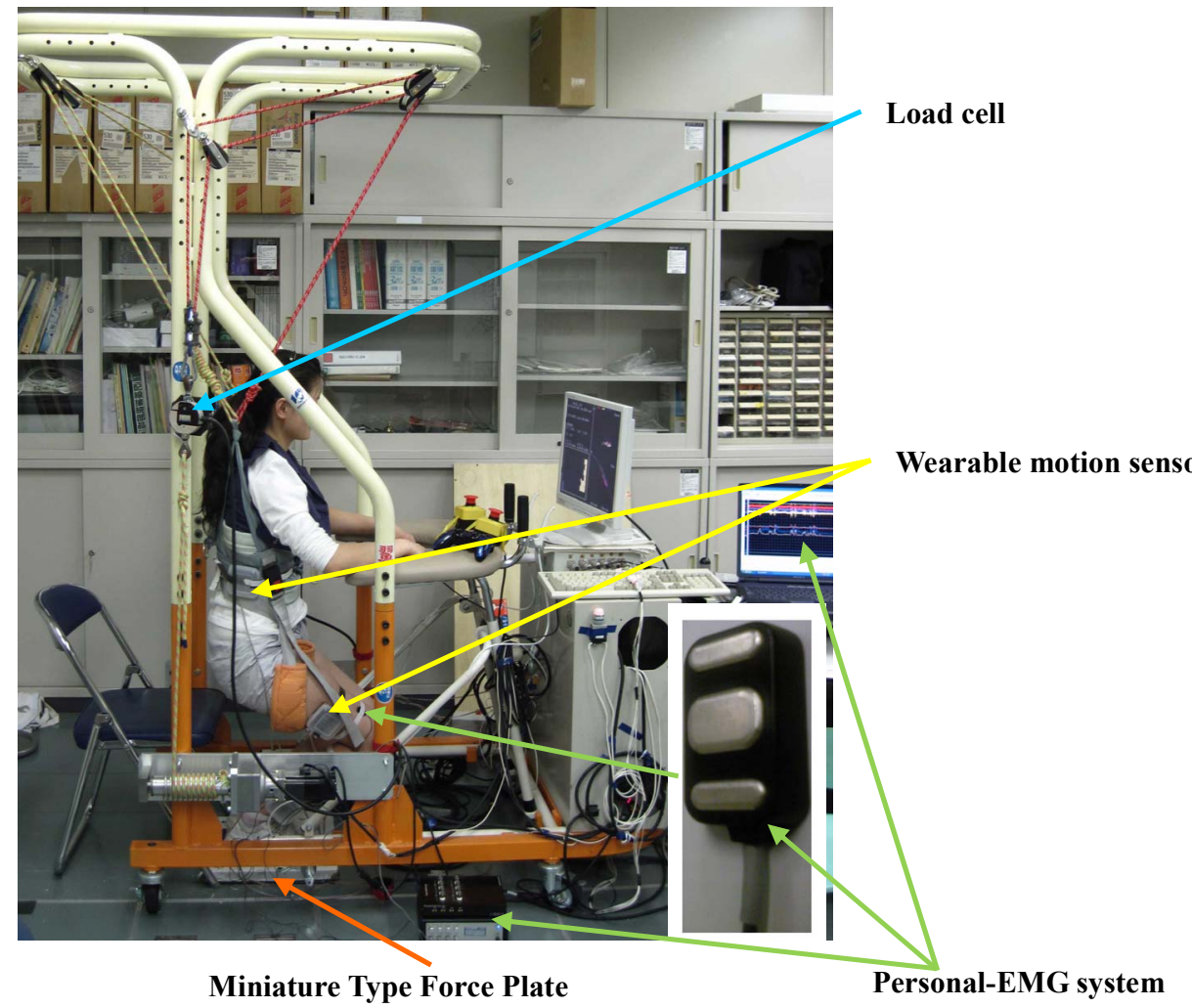

Fig. 5 Photograph of a subject in the standing-up experiment.

\subsection{Experimental Results}

As the complex kinetic system of standing-up process (SP) depends on a high degree of collaboration, in AnyBody Modeling System the muscle forces of lower limb were calculated based on a minimum fatigue criterion way. In the AnyBody process screenshots of Fig. 6, the muscle activation level could be identified by the muscle bulge level, the value and direction of GRF could be discerned by the length and direction of the arrow.

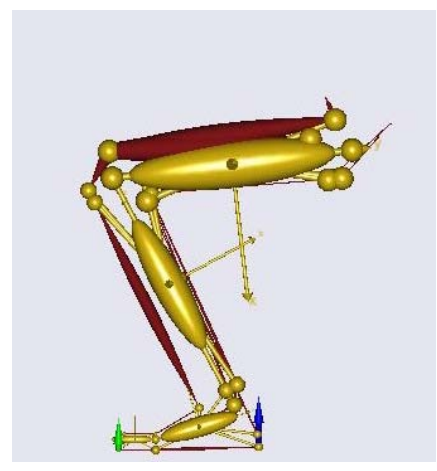

(a) $20 \%$ of standing-up process

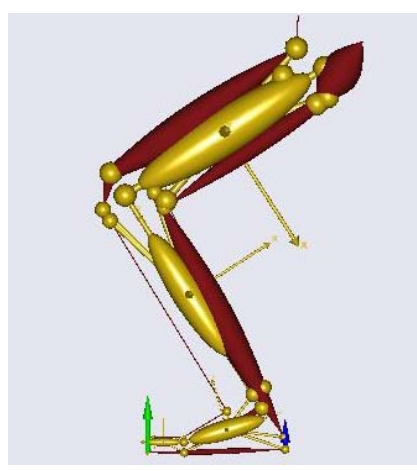

(b) $40 \%$ of standing-up process 


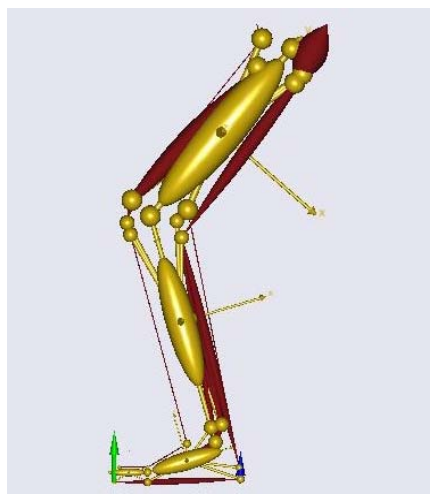

(c) $60 \%$ of standing-up process

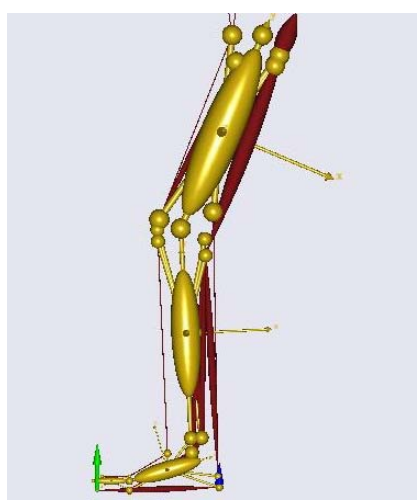

(d) $80 \%$ of standing-up process

Fig. 6. Screenshots of stand-up process video in AnyBody Modeling System, four postures in the process were shown.

The COP, GRF, and motion angles of body segments of four subjects are shown in Fig. 7, and the quantitative muscle force results of VM\&VL\&VI, AT, GAST, PL and SOL of four subjects are shown in Fig. 8. And in Fig. 8, in order to represent the law of muscle force variations clearly, the $X$-axis is chosen to show the percentage of SP while the $Y$-axis indicates the muscle force in units of Newton.

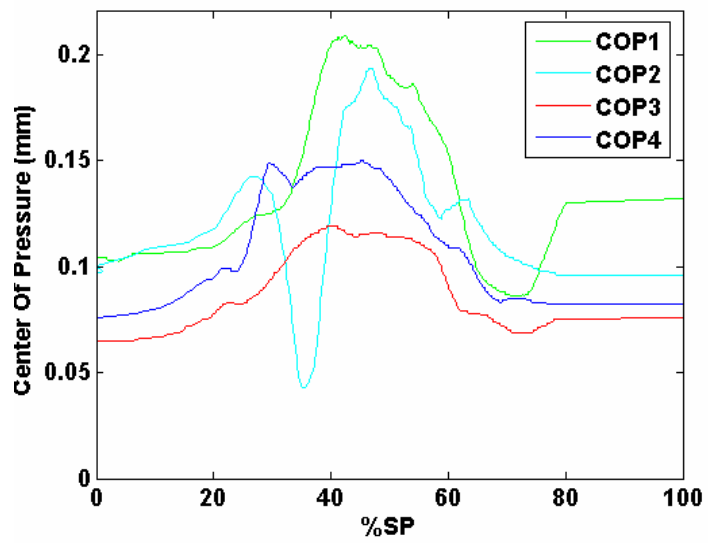

(a) COP of four subjects

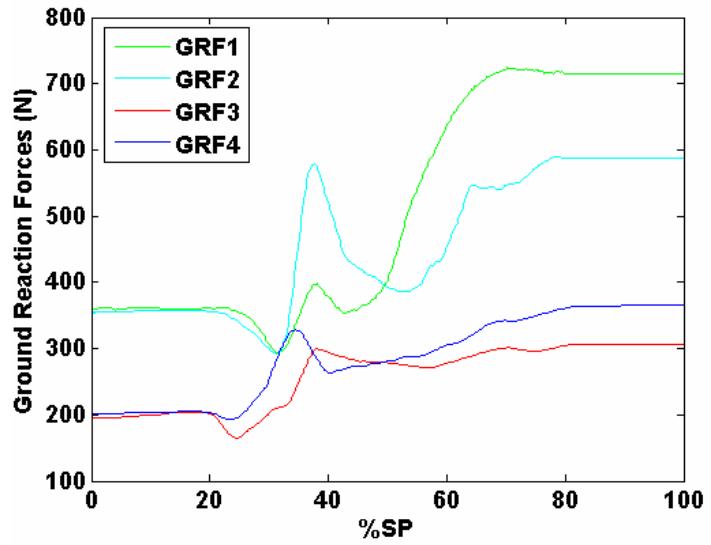

(b) GRF of four subjects 


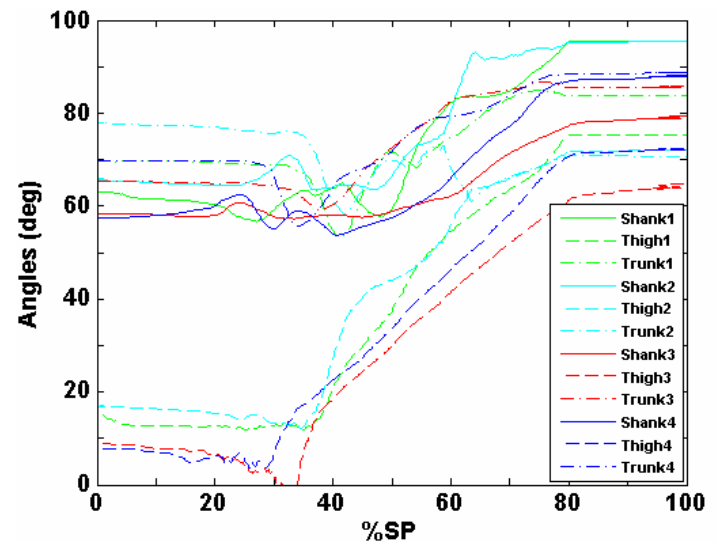

(c) Motion angles of four subjects

Fig. 7. COP, GRF and motion angle results of four subjects in impedance control method.

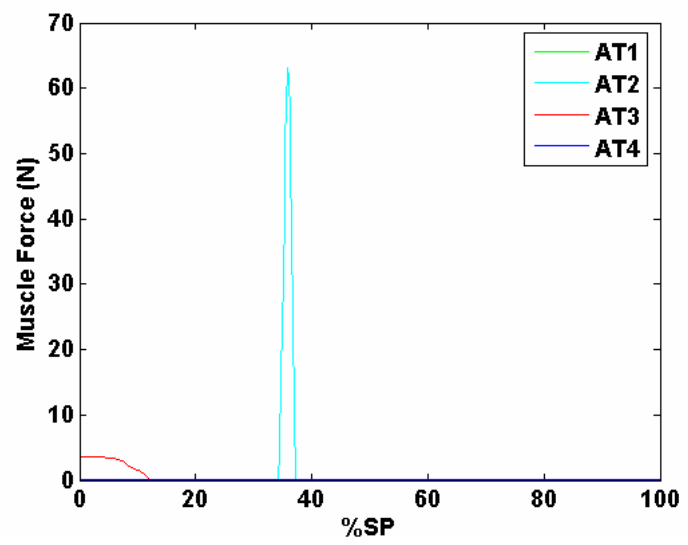

(a) Muscle forces of AT of four subjects

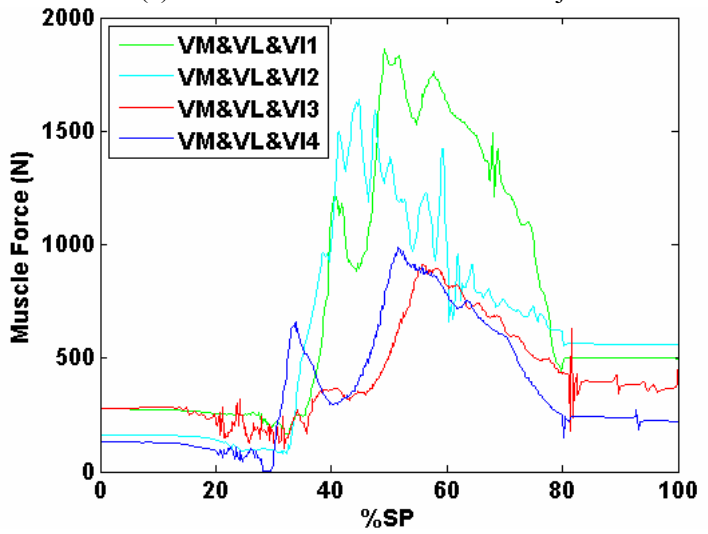

(b) Resultant muscle forces of VM\&VL\&VI of four subjects

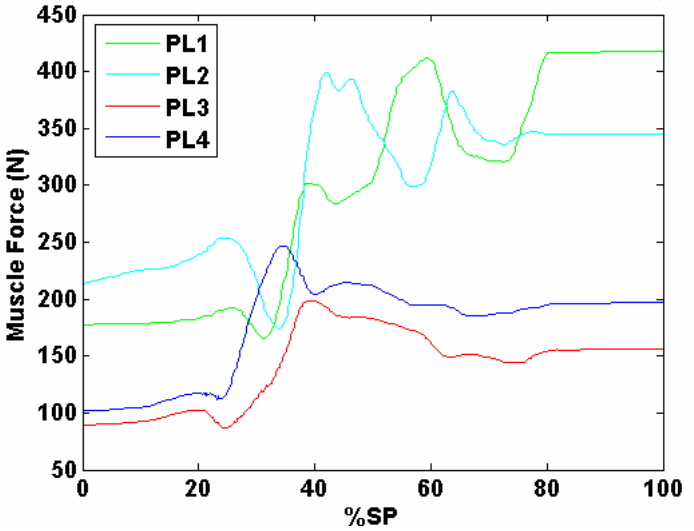

(c) Muscle forces of PL of four subjects 


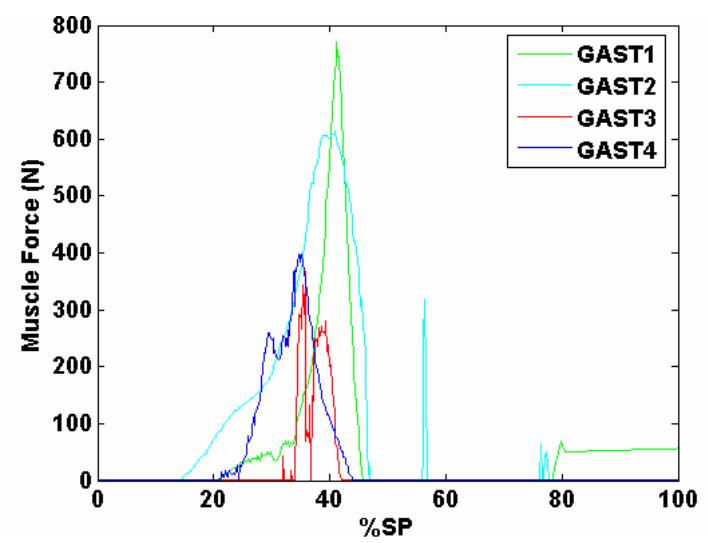

(d) Muscle forces of GAST of four subjects

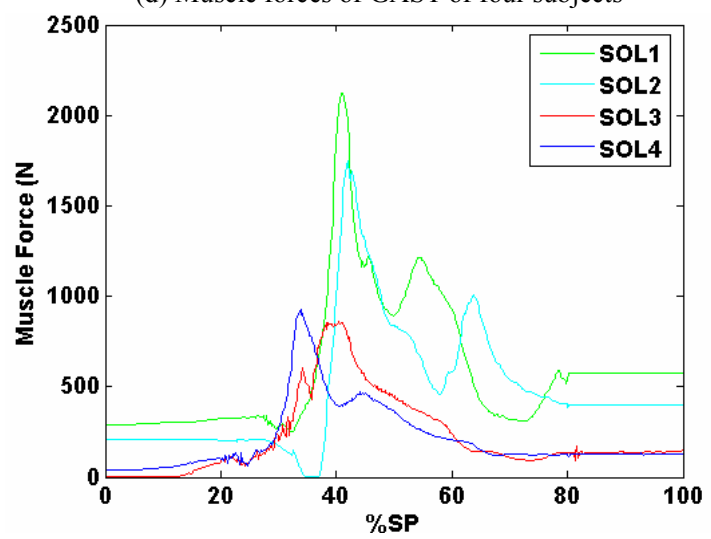

(e) Muscle forces of SOL of four subjects

Fig. 8. Muscle force results of four subjects in impedance control method.

The contrastive dynamic results of COP, GRF, motion angles, and contrastive muscle force results of VM\&VL\&VI, AT, GAST and SOL of one subject using own-standing method and in impedance control method were shown in Fig. 9 and Fig. 10 respectively. As the quantitative muscle force results were estimated based on an inverse dynamic method, the EMG results were used to do the validation. As shown in Fig. 10 (a1), (a2), (b1), (b2), (c1), (c2), (d1) and (d2), contradistinctive analysis was conducted between calculated AnyBody results and measured EMG results; the results of AnyBody Modeling System are drawn in red line while the results of EMG method are expressed in shadow. In comparison diagrams the $X$-axis represents percentage of SP, the left $Y$-axis indicates the muscle force in units of Newton, and the right $Y$-axis indicates the percentage of standard voluntary contraction of muscles.

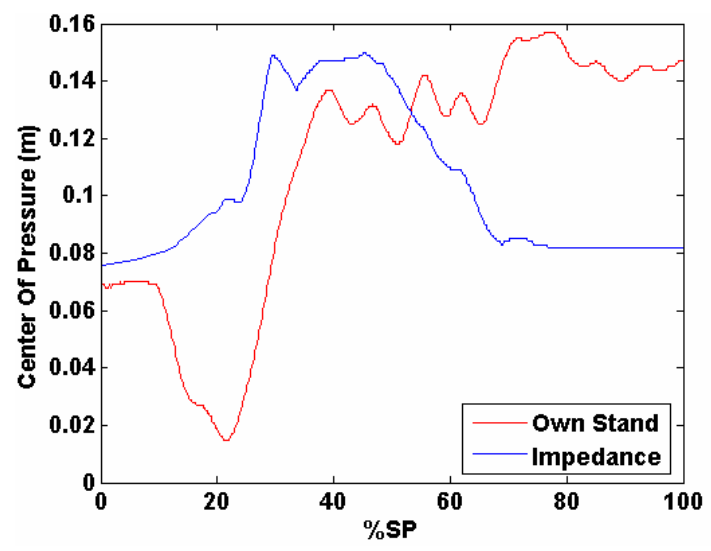

(a) Contradistinction of COP 


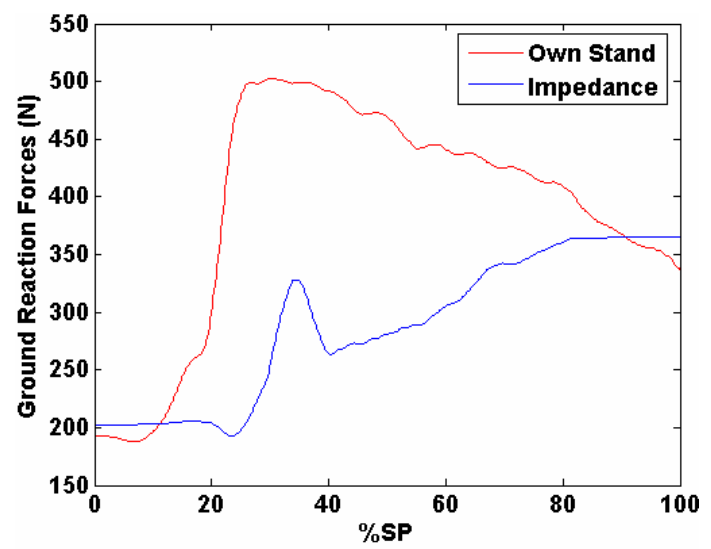

(b) Contradistinction of GRF

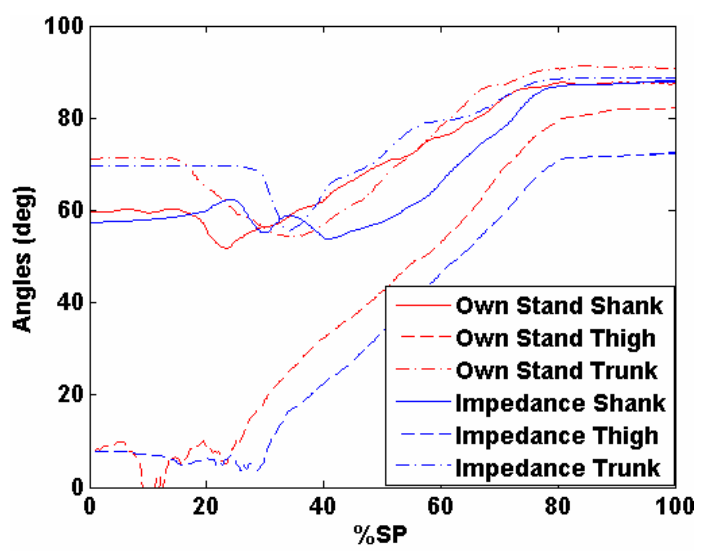

(c) Contradistinction of motion angles

Fig. 9. Contrastive COP, GRF and motion angle results between in own-standing method and in impedance control method.

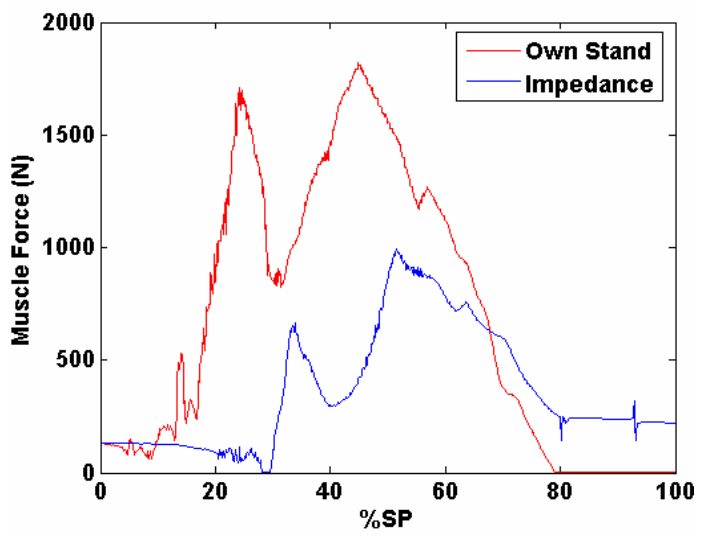

(a) Contradistinction of VM\&VL\&VI

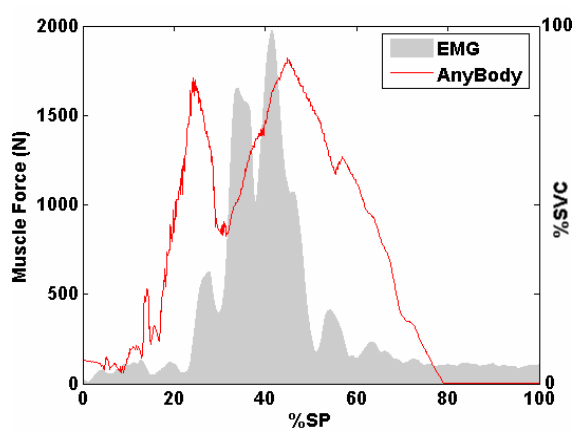

(a1) VM\&VL\&VI of own-standing method

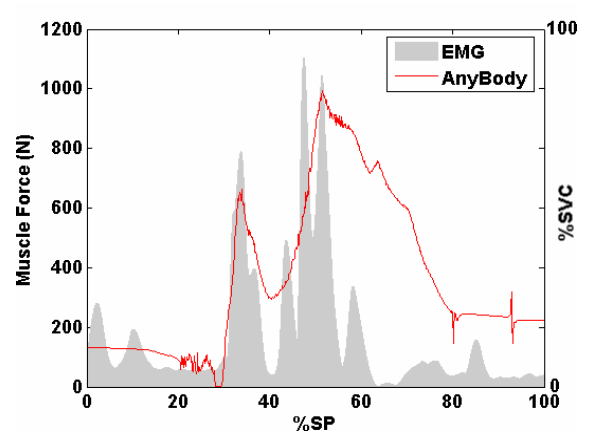

(a2) VM\&VL\&VI of impedance control method 


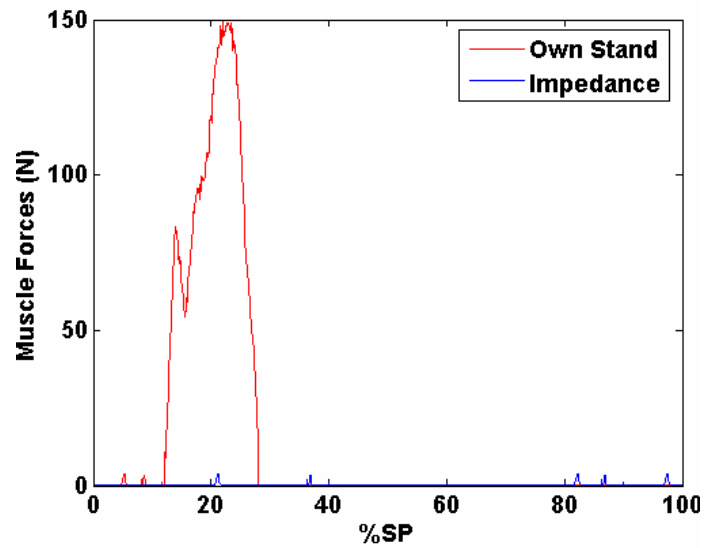

(b) Contradistinction of AT

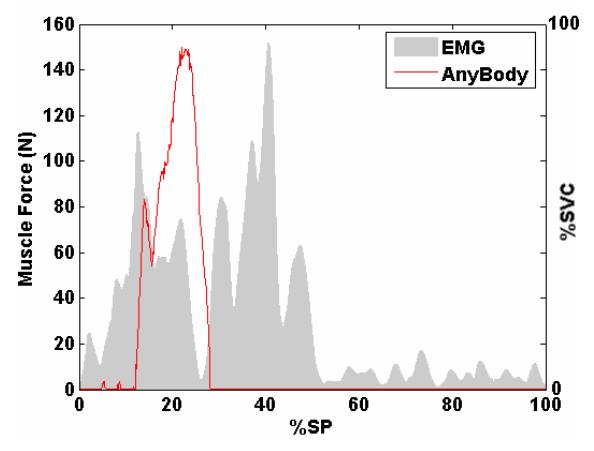

(b1) AT of own-standing method

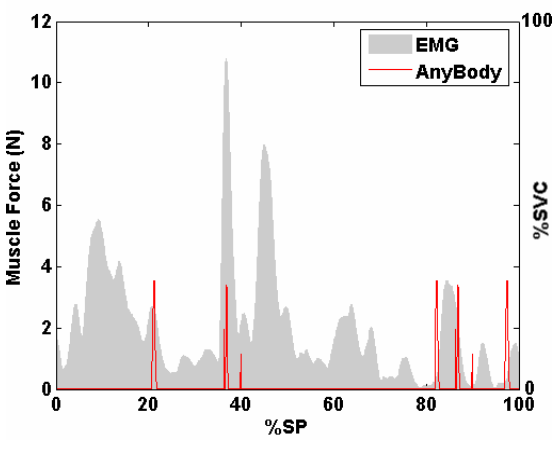

(b2) AT of impedance control method

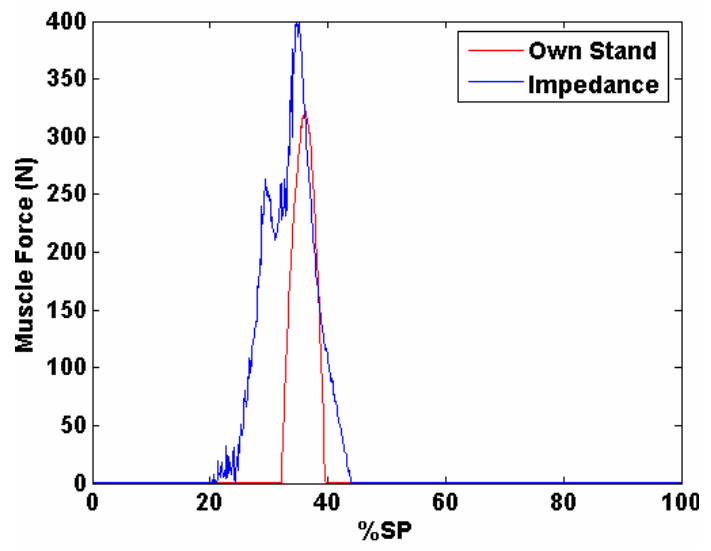

(c) Contradistinction of GAST

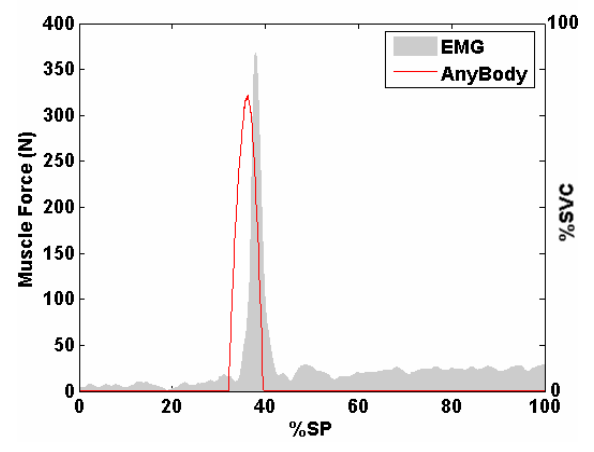

(c1) GAST of own-standing method

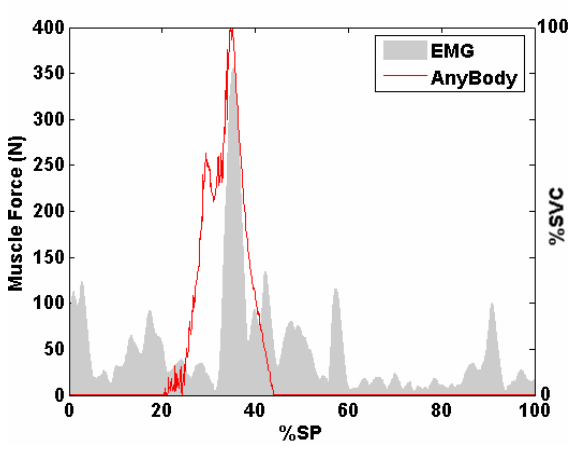

(c2) GAST of impedance control method 


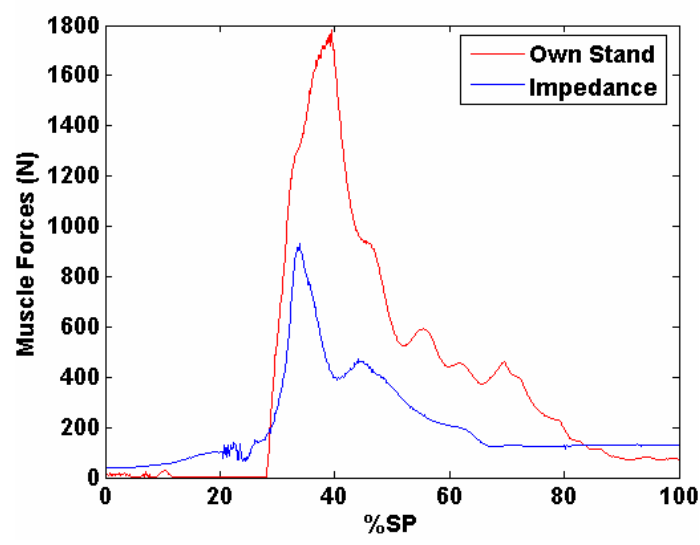

(d) Contradistinction of SOL

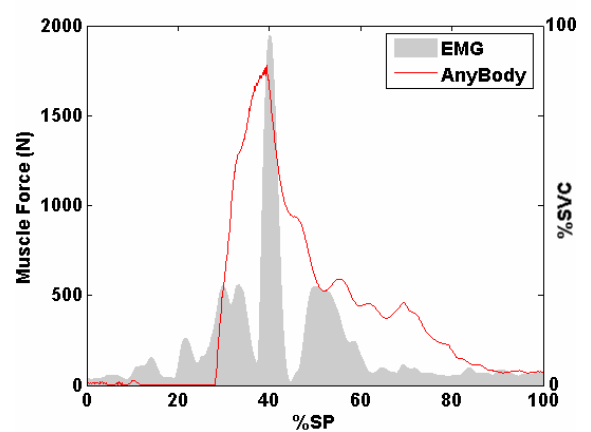

(d1) SOL of own-standing method

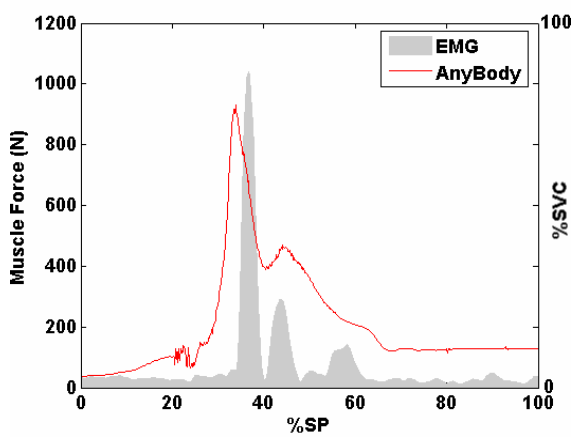

(d2) SOL of impedance control method

Fig. 10. Contrastive muscle force and EMG results between in own-standing method and in impedance control method.

\section{Discussions}

As shown in Fig. 7 and Fig. 8, experiment results of four subjects in one SP cycle were shown. As shown in Fig. 7 (a), COP started to increase from $20 \%$ of SP and decrease from $60 \%$ of SP, because the gravity center of human body always moves forward firstly and then backward when a healthy man stands up from a chair. As shown in Fig. 7 (b) and (c), the GRF, $\phi_{\text {thigh }}, \phi_{\text {shank }}$, and $\phi_{\text {foot all grew up continuously, but only vibrated from } 20 \% \text { to }}$ $60 \%$ of SP. This is because the subjects experienced more difficulty in this period and higher muscle forces were required. As shown in Fig. 8 (a), AT showed near-zero values but only a $60(\mathrm{~N})$ pulse at $38 \%$ of SP on subject 2 , that because the COP transformed to the posterior part of foot only in $38 \%$ of SP, and simultaneously, AT offered a force to balance the moment of the ankle joint. As shown in Fig. 8 (b), (c), (d) and (e), at $0 \%-20 \%$ of SP, all muscles showed relatively low values only to maintain balance in the preparation posture. At $20 \%-40 \%$ of SP, VL\&VM\&VI started to increase in parallel with the increases of motion angles of body segments, PL started to increase in parallel with the increase of GRF, and GAST and SOL showed obvious increases in parallel with the COP transformation from the middle part of the foot to anterior part of the foot. At $40 \%-60 \%$ of SP, VL\&VM\&VI continued growing up along with the increases of motion angles of body segments for pushing the human body moving upward, but GAST and SOL showed decrescendo values in parallel with the backward transformation of COP, moreover PL varied in parallel with the effect of GRF. At $60 \%-80 \%$ of SP, VL\&VM\&VI showed decrescendo values to complete the standing-up process, GAST and SOL showed vibrated and lower values because the COP vibrated in a relatively small range in this period. However, PL increased 
by the effect of increasing GRF and vibrated by the effect of vibrating COP. At $80 \%-100 \%$ of SP, the human body reached a stable standing posture and all muscle forces showed stable and lower values just to maintain the balance of the body. Therefore, it seems AT, GAST and SOL were mainly responsible for the movement balance of ankle joint, VL\&VM\&VI was mainly responsible for the upward movement of body trunk, PL was mainly responsible for the balance of human body. Together, they composed a cooperative muscle dynamic system in the SP.

As shown in Fig. 9 and Fig. 10, contrastive experiment results of one subject were shown between in own-standing method and in impedance control method. As shown in Fig. 9 (a) and (b), by the assistance effects of the rehabilitation robot, the COP were more stable, and the GRF showed conspicuous lower and more gradual increasing values in impedance control method than in own-standing method. And as shown in Fig. 9 (c), the variations of $\phi_{\text {thigh }}, \phi_{\text {shank, and }} \phi_{\text {foot }}$ showed similarities in the two experiment methods because the subject stood up along similar normal trajectories. As shown in Fig. 10 (a), (a1) and (a2), in the own-standing method, GRF appeared on the posterior part of foot at the beginning of SP. To balance the moment of ankle joint the force of AT increased and showed higher value in the $10 \%-30 \%$ of SP. However, in the impedance control method, AT was not activated because the GRF first appeared in the middle of the foot due to the effects of the rehabilitation robot. As shown in Fig. 10 (b), (b1) and (b2), VL\&VM\&VI showed lower and more hysteretic values in impedance control method than in own-standing method, because a previous upward movement of the body trunk was provided by the rehabilitation robot. As shown in Fig. 10 (c) and (d), GAST showed similar variation in the two control method. SOL, on the other hand, showed lower values in the impedance control method than in the own-standing method, because the COP transformation had smaller ranges in the impedance control method. Therefore, the rehabilitation robot could improve the dynamic conditions and decrease the muscle forces of lower limb effectively. Furthermore as shown in Fig. 10 (a1), (a2), (b1), (b2), (c1), (c2), (d1) and (d2), validation analysis was performed between the AnyBody method and the EMG method. Although the EMG muscle activation results were relative values in units of $\% \mathrm{SVC}$ while the AnyBody muscle force results were quantitative values in units of Newton, prominent similarity could be found in the contradistinction.

\section{Conclusions}

A quantitative approach for estimating muscle forces of lower limb based on inverse dynamics technology was developed. The rehabilitation robot provided effective assistance in the standing-up process, and the sensors performed effectively in measuring dynamic parameters of lower limbs. The measured data can be imported into the musculoskeletal model in AnyBody Modeling System. In the model the quantitative muscle forces were calculated through an inverse dynamics method, and the variation of the muscle force results showed practical sense in the SP. Furthermore, the EMG method was used for validation, and the muscle activation results of EMG method matched the muscle force results of AnyBody model. This approach appears to be practical for determining muscle force in musculoskeletal analysis of human lower limb.

\section{Acknowledgement}

The authors wish to acknowledge the support of the volunteer subjects of Robotics and Dynamics Research Lab in Kochi University of Technology. 


\section{References}

(1) Fent, T., Counting (On) An Aging Population, IEEE Computing in Science \& Engineering, Vol.8, No.6 (2006), pp.88-96.

(2) Mann, W.C., The Aging Population and Its Needs, IEEE Pervasive Computing, Vol.3, No.2 (2004), pp.12-14.

(3) Yoon, J., Novandy, B., Yoon, C. H., and Park, K. J., A 6-DOF Gait Rehabilitation Robot With Upper and Lower Limb Connections That Allows Walking Velocity Updates on Various Terrains, IEEE/ASME Trans. on Mechatronics, Vol.15, No.2 (2010), pp.201-215.

(4) Ferris, D. P., Sawicki, G. S., and Domingo, A., Powered lower limb orthoses for gait rehabilitation, Top Spinal Cord Inj Rehabil., Vol.11, No.2 (2005), pp.34-49.

(5) Hirata, R., Sakaki, T., Okada, S., Nakamoto, Z., Hiraki, N., Okajima, Y., Uchida, S., Tomita, Y., and Horiuchi, T., BRMS:Bio-Resposive Motion System (Rehabilitation System for Stroke Patients), Proc. of IEEE/RSJ Intl. Conf. on Intelligent Robots and Systems EPFL, Lausanne. Switzerland, Vol.2 (2002), pp.1344-1348.

(6) Merlo, A., Farina, D., Merletti, R., A Fast and Reliable Technique for Muscle Activity Detection From Surface EMG Signals, Transactions On Biomedical Engineering, Vol.50, No.3(2003). pp.316-323.

(7) Su, Y., Fisher, M.H., Wolczowski, A., Bell, G.D., Burn, D.J., Gao, R.X., Towards an EMG-Controlled Prosthetic Hand Using a 3-D Electromagnetic Positioning System, IEEE Transactions on Instrumentation And Measurement, Vol.56, No.1(2007). pp.178-186.

(8) Hou, Y.F., Zurada, J.M., Karwowski, W., Marras, W.S., Davis, K., Estimation of the Dynamic Spinal Forces Using a Recurrent Fuzzy Neural Network, IEEE Transactions on Systems, Man, and Cybernetics—Part B: Cybernetics, Vol.37, No.1(2007). pp.100-109.

(9) Bogey, R.A., Perry, J., Gitter, A. J., An EMG-To-Force Processing Approach For Determining Ankle Muscle Forces During Normal Human Gait, IEEE Transactions on Neural Systems and Rehabilitation Engineering, Vol.13, No.3(2005), pp.302-310.

(10) Aeberhard, M., Michellod, Y., Mullhaupt, P., Terrier, A., Pioletti, D.P., Gillet, D., Dynamical Biomechanical Model of the Shoulder: Null Space Based Optimization of the Overactuated System, Proceedings of IEEE International Conference on Robotics and Biomimetics, Bangkok. Thailand, No.4912981(2009-2), pp.67-73.

(11) Anderson, S.R., Lepora, N.F., Porrill, J., Dean, P., Nonlinear Dynamic Modeling of Isometric Force Production in Primate Eye Muscle, IEEE Transactions on Biomedical Engineering, Vol.57, No.7(2010), pp.1554-1567.

(12) Lee, S.W., Kamper, D.G.., Modeling of Multiarticular Muscles: Importance of Inclusion of Tendon-Pulley Interactions in the Finger, IEEE Transactions on Biomedical Engineering, VOL.56, No.9(2009), pp.2253-2262.

(13) Ferreira, J.P., Crisostomo, M.M., Coimbra, A.P., Human Gait Acquisition and Characterization, IEEE Transactions on Instrumentation and Measurement, Vol.58, No.9(2009), pp.2979-2988.

(14) Liu, K., Liu, T., Shibata, K., Inoue, Y., Zheng, R.C., Novel Approach to Ambulatory Assessment of Human Segmental Orientation on A Wearable Sensor System, Journal of Biomechanics., Vol.42, No.16(2009), pp.2747-2752.

(15) Luinge, H.J., Veltink, P.H., Measuring Orientation of Human Body Segments Using Miniature Gyroscopes and Accelerometers, Med. Biol. Eng. Comput., Vol.43, No.2(2005), pp.273-282.

(16) Boonstra, M.C., Slikke, R.M., Keijsers, N.L., Lummel, R.C., Malefijt, M.C., Verdonschot, N., The Accuracy of Measuring the Kinematics of Rising from a Chair with Accelerometers and Gyroscopes, Journal of Biomechanics, Vol.39, No.2(2006), pp.354-358. 
(17) Parasuraman, S., Yee, K.C., Oyong, A., Human upper limb and arm kinematics for robot based rehabilitation, IEEE/ASME International Conference on Advanced Intelligent Mechatronics, Singapore, No.5229906(2009-7), pp.845-850.

(18) Urban, M., and Bajcsy, P., Fusion of Voice, Gesture, and Human-Computer Interface Controls for Remotely Operated Robot, 7th International Conference on Information Fusion (FUSION), Vol.2 (2005-7), pp.1644-1651.

(19) McMinn, R.M.H., Hutchings, R.T., Logan, B.M., A Colour Atlas of Foot and Ankle Anatomy, (1982), p.10-21, Wolfe Medical Publications Ltd.

(20) Perry, J., Ireland, M., Gronley, J., Hoffer, M., Predictive Value of Manual Muscle Testing and Gait Analysis in Normal Ankles by Electromyography, Foot Ankle, Vol.6, No.5(1986), pp.254-259. 